\title{
Sliding-mode Trajectory Tracking Control of Autonomous Surface Vessel
}

\author{
Kui LIANG \\ College of Electrical \\ Guangxi University \\ Nanning, Guangxi, China \\ E-mail: 1216231989@qq.com \\ Zengqiang LEI \\ College of Electrical \\ Guangxi University \\ Nanning, Guangxi, China \\ E-mail: 604723614@qq.com
}

\author{
Jiaoyan $\mathrm{AI}^{*}$ \\ College of Electrical \\ Guangxi University \\ Nanning, Guangxi, China \\ E-mail: shinin@vip.163.com \\ +* Corresponding author
}

\author{
Gang LIU \\ College of Electrical \\ Guangxi University \\ Nanning, Guangxi, China \\ E-mail: liugang5000@163.com
}

\begin{abstract}
In this paper, The kinematics and dynamics model of autonomous surface vessels (ASV) is established for the trajectory tracking control of ASV. A trajectory tracking control law is proposed by the sliding mode variable structure control method. Through theoretic analysis, the control law can guarantee the asymptotic stability of the trajectory tracking of ASV. The simulation results show that the proposed method is effective.
\end{abstract}

\section{Keywords -ASV; sliding-mode; trajectory; tracking control}

\section{INTRODUCTION}

With two actuator inputs, autonomous surface vessels (ASV) are generally considered as vehicles since they posse three degrees freedom. Tracking control of under-actuated vehicles has received wide attention scholars. For ASV, tracking trajectory is difficult to implement since the system has underactuated characteristics and the model is highly nonlinear. Most of the current studies focus on output feedback linearization [1-2], backstepping [3], Lyapunov direct method [4] and sliding mode control.

According to the nonlinear dynamics model and the kinematic model of ASV, a method based on sliding mode control is proposed to solve the trajectory tracking problem of ASV. We use two sliding surfaces for calculation of the propeller forces. The first sliding surface is a first-order surface in terms of the surge-velocity tracking errors and the second sliding surface is the first-order yaw angular velocity tracking errors. By measuring the absolute position and orientation of the ship, the surge, sway and yaw angular velocities are calculated. The stability of the trajectory tracking is proved by using Lyapunov's theorem, and the validity of the proposed control method is verified by theoretical analysis and simulation experiments.

\section{MODEL}

This paper studies the ASV motion diagram shown in Fig. 1.The model has two actuators that enable the ASV to move surge, laterally and yaw. Assume that ASV motion satisfies the following conditions:

1) neglect the influence of environmental disturbance caused by wind, wave and flow;

2) neglect the ASV's heave, pitch and roll, only consider the motion in the horizontal plane of ASV;

3) the ASV mass distribution is even. The kinematics of three-DOF underactuated ASV is as figure 1.

$$
\left[\begin{array}{c}
\dot{\mathrm{x}} \\
\dot{y} \\
\dot{\psi}
\end{array}\right]=\left[\begin{array}{ccc}
\cos \psi & -\sin \psi & 0 \\
\sin \psi & \cos \psi & 0 \\
0 & 0 & 1
\end{array}\right]\left[\begin{array}{l}
u \\
\mathrm{v} \\
r
\end{array}\right]
$$

where $x$ and $y$ denote the position of the center of mass , $\psi$ is the orientation angle of the vessel in the inertial inertial reference frame and $u, v, r$ represent the surge, lateral, and angular velocity of the ASV in the body-fixed frame.

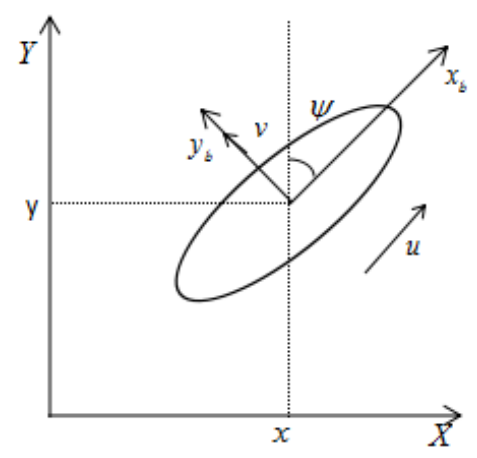

Figure 1. Planar model of a surface vessel

A simplified model of the dynamics of ASV is a nonlinear equation of motion in the body-fixed frame as 


$$
\begin{aligned}
& \dot{u}=\frac{\mathrm{m}_{22}}{m_{11}} v r-\frac{d_{11}}{m_{11}} u+\frac{\tau_{u}}{m_{11}} \\
& \dot{v}=-\frac{\mathrm{m}_{11}}{m_{22}} \mathrm{u} r-\frac{d_{22}}{m_{22}} v \\
& \dot{r}=\frac{\mathrm{m}_{11}-m_{22}}{m_{33}} u v-\frac{d_{33}}{m_{33}} r+\frac{\tau_{r}}{m_{33}}
\end{aligned}
$$

The general spatial equations for ASV can be found in [5].In(2), ASV have only two inputs, as the surge control force $\tau_{u}$ and the yaw control moment $\tau_{\mathrm{r}}$.The parameters $m_{i i}$ and $d_{i i}$ are the components of the ship inertial and damping parameter matrix in the body-fixed frame.

The model velocity errors are given by

$$
u_{e}=u-u_{d}, \quad r_{e}=r-r_{d}
$$

The $u_{d}$ and $r_{d}$ are the expected value, $u_{e}$ and $r_{e}$ the error, $u$ and $r$ are the actual value.

\section{SLIDING Mode Control LAW}

Sliding mode variable structure control [2] is a kind of discontinuous control method, which can force the system to move to the equilibrium point along the specified sliding surface. It has good self-adaptability to external disturbance and parameter uncertainty.

In the sliding mode control method, we first need to define the asymptotic stabilizing sliding surface(S) of the tracking error function so that all state trajectories reach the sliding surface in finite time and move along the sliding surface $(S=0)$ until convergence to equilibrium point. A Lyapunov function as

$$
V=\frac{1}{2} S^{T} S
$$

If the time derivative is negative, then the system satisfies the Lyapunov stability theory. For the ASV, two sliding surfaces are defined to determine the control input, so the reach-ability condition of each sliding mode is [6]

$$
S_{i} \dot{S}_{i} \leq-\eta_{i}\left|S_{i}\right| \quad \eta_{i}>0, i=1,2
$$

which $\eta_{i}$ is a positive constant.

\section{A. Surge Control Law}

The sliding surface is a first-order surface in terms of the surge-velocity tracking errors

$$
S_{1}=u_{e}+\lambda_{1} \int_{0}^{t} u_{e}(\tau) d \tau, \quad \lambda_{1}>0
$$

design of surge sliding mode control law:

$$
\begin{aligned}
& \tau_{u}=\hat{\tau}_{u}-k_{1} \operatorname{sat}\left(S_{1} / \phi\right) \\
& \hat{\tau}_{u}=-\hat{\mathrm{m}}_{22} v r+\hat{d}_{11} u-\hat{\mathrm{m}}_{11}\left(-\dot{u}_{d}+\lambda_{1} u_{e}\right)
\end{aligned}
$$

the symbol “ $\wedge$ ”is used to the estimated model parameters. In order to improve the system, we define an approximate sliding-mode control law using a high-slope saturation function as in [7]

$$
\operatorname{sat}(S / \phi)=\left\{\begin{array}{c}
S / \phi,|S| \leq \phi \\
\operatorname{sgn}(S),|S|>\phi
\end{array}\right.
$$

where $\phi$ is a positive constant which defines a small boundary layer around the surface.

In order to determine $k_{1}$, define uncertain boundary of model parameters and environmental interference in (2) as

$$
\left|m_{i i}-\hat{m}_{i i}\right| \leq M_{i i} \quad\left|d_{i i}-\hat{d}_{i i}\right| \leq D_{i i} \quad i=1,2,3
$$

Define a Lyapunov candidate function

$$
V_{1}=\frac{1}{2} m_{11} S_{1}^{2}
$$

The time derivative of (10) can be derived

$$
\begin{aligned}
& \begin{aligned}
\dot{\mathrm{V}}_{1}= & \frac{1}{2} \mathrm{~m}_{11} \mathrm{~S}_{1} \dot{\mathrm{S}}_{1} \\
= & \mathrm{S}_{1}\left[\left(m_{22}-\hat{m}_{22}\right) v r+\left(\hat{d}_{11}-d_{11}\right) u\right. \\
& +\left(m_{11}-\hat{m}_{11}\right)\left(-\dot{u}_{d}+\lambda_{1} u_{e}\right) \\
& \left.-k_{1} \operatorname{sat}\left(S_{1} / \phi_{1}\right)\right]
\end{aligned} \\
& \boldsymbol{k}_{1}=\mathbf{M}_{2}|v r|+D_{1}|u|
\end{aligned}
$$
reach condition is achieved:

$$
\dot{V}_{1}=m_{11} S_{1} \dot{S}_{1} \leq-\hat{m}_{11} \eta_{1}\left|S_{1}\right|
$$

\section{B. Angular Control Law}

We define the sliding surface of the vessel's angular motion tracking errors 


$$
S_{2}=r_{e}+\lambda_{2} \int_{0}^{t} r_{e}(\tau) d \tau, \quad \lambda_{2}>0
$$

design of angular sliding mode control law:

$$
\tau_{r}=\hat{\tau}_{r}-k_{2} \operatorname{sat}\left(S_{2} / \phi_{2}\right)
$$

which $\hat{\tau}_{u}=\hat{\mathrm{m}}_{33}\left(\dot{r}_{d}-\lambda_{2} r_{e}\right)+\hat{d}_{33} r-\left(\hat{\mathrm{m}}_{11}-\hat{\mathrm{m}}_{22}\right) u v$ define a Lyapunov candidate function.

$$
V_{2}=\frac{1}{2} m_{33} S_{2}
$$

The time derivative of (15) can be derived

$$
\begin{aligned}
\dot{\mathrm{V}}_{2} & =\mathrm{m}_{33} S_{2} \dot{S}_{2} \\
& =S_{2}\left[\left(\left(m_{11}-\hat{m}_{11}\right)-\left(m_{22}-\hat{m}_{22}\right)\right) u v\right. \\
& +\left(\hat{d}_{33}-d_{33}\right) r+\left(m_{33}-\hat{m}_{33}\right)\left(-\dot{r}_{d}+\lambda_{2} r_{e}\right) \\
& \left.-k_{2} \operatorname{sat}\left(S_{2} / \phi_{2}\right)\right] \\
\text { If } & k_{2}=\left(\mathrm{M}_{11}-\mathrm{M}_{2}\right)|u v|+D_{3} r, \text { hence, the following } \\
& +M_{33}\left|-\dot{r}_{d}+\lambda_{2} r_{e}\right|+\hat{m}_{33} \eta_{2}
\end{aligned}
$$
reach condition is achieved:

$$
\dot{V}_{1}=\mathrm{m}_{33} S_{2} \dot{S}_{2} \leq-\hat{m}_{33} \eta_{2}\left|S_{2}\right|
$$

\section{Simulation And Results Analysis}

In order to verify the effectiveness of the proposed control law, the following numerical simulation example of a surface vessel model with two independent propeller, the ship model specific parameters are as follows [8]

$$
\begin{array}{ll}
\hat{m}_{11}=200 \mathrm{~kg}, & \hat{m}_{22}=250 \mathrm{~kg}, \quad \hat{m}_{33}=80 \mathrm{~kg}, \\
\hat{d}_{11}=70 \mathrm{~kg} / \mathrm{s}, & \hat{d}_{22}=200 \mathrm{~kg} / \mathrm{s}, \hat{d}_{33}=200 \mathrm{~kg} / \mathrm{s}, \\
\tau_{u}=200 \mathrm{~N}, & \tau_{r}=50 \mathrm{~N} \cdot \mathrm{m}
\end{array}
$$

We use reference ellipse trajectory in (18) and initial conditions are $\mathrm{x}=15 \mathrm{~m}, y=0 \mathrm{~m}, \psi=30^{\circ}$, the initial velocity are $\mathrm{u}=0.5 \mathrm{~m} / \mathrm{s}, v=r=0 \mathrm{~m} / \mathrm{s}$ and control parameters

$$
\begin{aligned}
& \lambda_{1}=0.2, \quad \lambda_{2}=0.5, \quad \eta_{1}=0.1, \quad \eta_{2}=0.1 \\
& \phi_{1}=\phi_{2}=0.1
\end{aligned}
$$

$$
\left\{\begin{array}{l}
\mathrm{x}_{d}=10 \sin 0.02 t \\
\mathrm{y}_{d}=10 \cos 0.02 t
\end{array}\right.
$$

The simulation results are shown in Fig. The ASV quickly tracks the desired trajectory and moves along the ellipse.

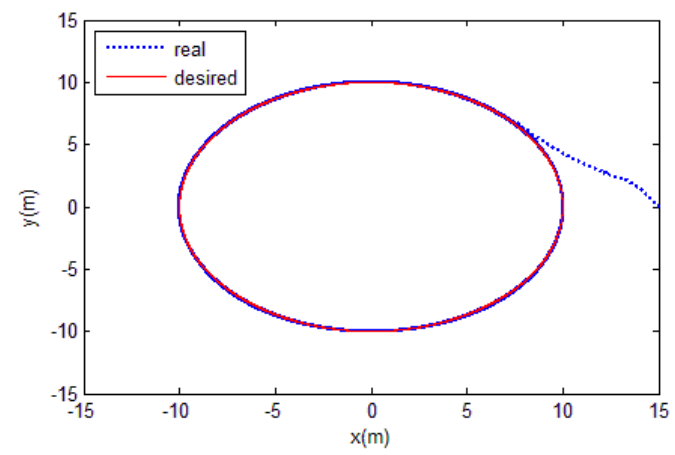

Figure 2. Trajectory of the model vessel in $x-y$ plane

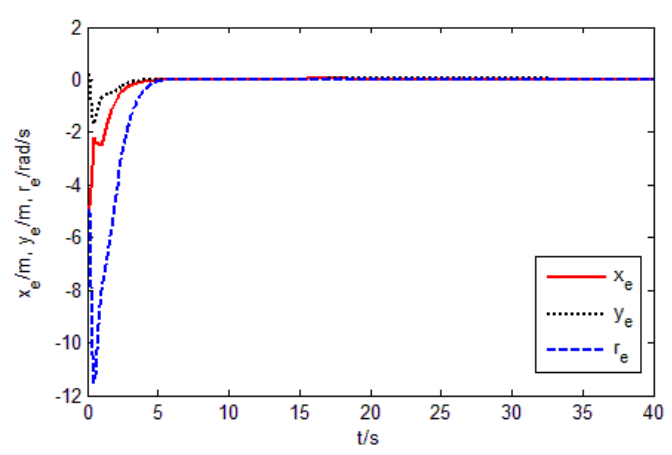

Figure 3. The error of velocities

Fig 2 displays the course of vessel trajectory tracking under the sliding control law. It is clearly seen that the vessel follows the reference trajectory with high accuracy and has fast tracking speed. Fig 3 shows that the tracking errors simultaneously convergent to zero with fast convergence rate. It verify the effectiveness of the proposed control law.

\section{CONCLUSION}

In this paper, a sliding mode control law is proposed to solve the trajectory tracking problem of an underactuated surface vessel with only a surge force and a yaw moment. By using a first-order sliding surface in the terms of surge tracking errors and a first-order sliding surface in the terms of angular velocity tracking errors, we obtain the input control law. Ellipse trajectory tracking example demonstrates the effectiveness of the suggested approach.

\section{ACKNOWLEDGEMENT}

This research was financially supported by National Nature Science Foundation of China (61563002) and Guangxi Scientific and Technological Project Fund (14251009).The corresponding author of this paper is Ai Jiaoyan, a professor at Guangxi University.

\section{REFERENCES}

[1] Pettersen K, Nijmeijer $\mathrm{H}$. Output feedback tracking control for ships[J]. New Directions in Nonlinear Observer Design, 1999, 20:311-334. 
[2] Wondergem M, Lefeber E, Pettersen K Y, et al.Output feedback tracking of ships[J]. Control Systems Technology, IEEE Transactions on, 2011.

[3] Godhavn,Fossen TI, Berge S.Non-linear and adaptive backstepping designs for tracking control of ship[J]. International Journal of Adaptive Control and Signal Processing, 1998.

[4] Do K D, Pan J.Underactuated ship global tracking without measurement of velocities[C]. In American Control Conference, Proceedings of the 2003, 2003:2012-2017.
[5] Fossen.T.I. Marine control systems guidance navigation and control of ships rigs and underwater vehicle[M]. Trondheim,Norway: Marine Cybernetics, 2002.

[6] Ashrafiuon H. Mushe K.RMcninch L, Soltan R.A. Sliding model tracking control of surface vessels[J]. IEEE Transactions on Industrial Eletronics, 2008.

[7] Khalil H K.Nonlinear system[M].3ed. Upper Saddle River,NJ:Prentice-Hall,2002.

[8] M.Rayhanoglu, Exponential stabilization of an underactuated autonomous $\quad$ surface vessel[J], Automatica, vol.33,no.6.pp.2249-2254,1997. 